TNF- $\alpha$-induced upregulation of p100 is somehow blocked under inflammatory conditions, such as in that in RA synovial joints, or do pathologic conditions induce signaling molecule(s) that suppress the inhibitory effect of p100? Second, while p100 acts as an I B-like molecule that binds to RelB, p100 also binds to the RelA/ p50 dimer $(11,18)$. Given that Rela ${ }^{--}$or Relb $^{-/}$single-knockout mice do not show an altered bone phenotype, p100 may suppress TNF- $\alpha$-induced osteoclast formation by retaining both RelA/p50 complex and RelB in the cytoplasm. Thus, it will be interesting to investigate the bone phenotype of Rela $^{-1-}$ Relb $^{-/-}$mice. Third, the mechanism of how TNF- $\alpha$ suppresses the degradation of TRAF3 has not been clarified. It will also be intriguing to test whether TNF- $\alpha$-induced osteoclast formation is enhanced in $\mathrm{Traf3}^{-/-}$ mice. Given that Traf3 $3^{-/-}$mice die soon after birth (14), transfer of Traf3 $^{-/-}$bone marrow cells to WT mice would be a feasible way to investigate the role of TRAF3 in TNF- $\alpha$ induced osteoclast formation in vivo. Finally, what is the role of bone-forming osteoblasts in TNF- $\alpha$-induced pathologic bone resorption, since TNF- $\alpha$ is known to induce RANKL expression in osteoblasts? These intriguing questions remain for future study.

In conclusion, Yao et al. (13) have convincingly demonstrated that NF-кB2p100 plays a negative role in suppressing TNF- $\alpha-$ induced osteoclast formation under pathologic conditions using various animal models. Thus, blockade of the processing of p100 might be a novel strategy to treat various bone diseases such as RA, in which TNF- $\alpha$-induced osteoclast formation plays a crucial role in the progression of diseases.

Address correspondence to: Sakae Tanaka, Department of Orthopaedic Surgery, Faculty of Medicine, The University of Tokyo, 7-3-1 Hongo, Bunkyo-ku, Tokyo 113-0033, Japan. Phone: 81-3-3815-5411, ext. 33376; Fax: 81-3-3818-4082; E-mail: tanakas-ort@ h.u-tokyo.ac.jp.

1. Tanaka, S., Nakamura, K., Takahasi, N., and Suda, T. 2005. Role of RANKL in physiological and pathological bone resorption and therapeutics targeting the RANKL-RANK signaling system. Immunol. Rev. 208:30-49.

2. Theill, L.E., Boyle, W.J., and Penninger, J.M. 2002. RANK-L and RANK: T cells, bone loss, and mammalian evolution. Annu. Rev. Immunol. 20:795-823.

3. Scott, D.L., and Kingsley, G.H. 2006. Tumor necrosis factor inhibitors for rheumatoid arthritis. N. Engl. J. Med. 355:704-712.

4. Azuma, Y., Kaji, K., Katogi, R., Takeshita, S., and Kudo, A. 2000. Tumor necrosis factor-alpha induces differentiation of and bone resorption by osteoclasts. J. Biol. Chem. 275:4858-4864.

5. Kim, N., et al. 2005. Osteoclast differentiation independent of the TRANCE-RANK-TRAF6 axis. J. Exp. Med. 202:589-595.

6. Kobayashi, K., et al. 2000. Tumor necrosis factor alpha stimulates osteoclast differentiation by a mechanism independent of the ODF/RANKL-
RANK interaction. J. Exp. Med. 191:275-286.

7. Li, J., et al. 2000. RANK is the intrinsic hematopoietic cell surface receptor that controls osteoclastogenesis and regulation of bone mass and calcium metabolism. Proc. Natl. Acad. Sci. U. S. A. 97:1566-1571.

8. Bonizzi, G., and Karin, M. 2004. The two NF-kap$\mathrm{paB}$ activation pathways and their role in innate and adaptive immunity. Trends Immunol. 25:280-288.

9. Franzoso, G., et al. 1997. Requirement for NF-kap$\mathrm{paB}$ in osteoclast and B-cell development. Genes Dev. 11:3482-3496.

10. Iotsova, V., et al. 1997. Osteopetrosis in mice lacking NF-kappaB1 and NF-kappaB2. Nat. Med. 3:1285-1289.

11. Novack, D.V., et al. 2003. The IkappaB function of NF-kappaB2 p100 controls stimulated osteoclastogenesis. J. Exp. Med. 198:771-781.

12. Vaira, S., et al. 2008. RelB is the NF-kappaB subunit downstream of NIK responsible for osteoclast differentiation. Proc. Natl. Acad. Sci. U. S. A. 105:3897-3902.

13. Yao, Z., Xing, L., and Boyce, B.F. 2009. NF-кB p100 limits TNF-induced bone resorption in mice by a TRAF3-dependent mechanism. J. Clin. Invest. 119:3024-3034.

14. He, J.Q., et al. 2006. Rescue of TRAF3-null mice by p100 NF-kappa B deficiency. J. Exp. Med. 203:2413-2418.

15. Liao, G., Zhang, M., Harhaj, E.W., and Sun, S.C. 2004. Regulation of the NF-kappaB-inducing kinase by tumor necrosis factor receptor-associated factor 3-induced degradation. J. Biol. Chem. 279:26243-26250.

16. Skaug, B., Jiang, X., and Chen, Z.J. 2009. The role of ubiquitin in NF-kappaB regulatory pathways. Annu. Rev. Biochem. 78:769-796.

17. Dejardin, E., et al. 2002. The lymphotoxin-beta receptor induces different patterns of gene expression via two NF-kappaB pathways. Immunity. 17:525-535.

18. Basak, S., et al. 2007. A fourth IkappaB protein within the NF-kappaB signaling module. Cell. 128:369-381.

\title{
Pathogenic antibodies are active participants in spinal cord injury
}

\author{
Gregory A. Dekaban and Sakina Thawer
}

BioTherapeutics Research Laboratory, Molecular Brain Research Group, Robarts Research Institute and Department of Microbiology and Immunology, The University of Western Ontario, London, Ontario, Canada.

\begin{abstract}
The role of B cells and autoimmunity as contributing factors to poor neurological outcomes following spinal cord injury (SCI) is poorly understood. The study by Ankeny et al., in this issue of the JCI, identifies a new immunopathological mechanism arising after SCI in mice (see the related article beginning on page 2990). The study shows that $B$ cells produce pathogenic antibodies that impair lesion repair, resulting in worse neurological outcome. This new understanding of SCI disease pathogenesis, if confirmed in humans, reveals potential avenues for the development of novel neuroprotective immunotherapies.
\end{abstract}

Conflict of interest: The authors have declared that no conflict of interest exists.

Citation for this article: J. Clin. Invest. 119:2881-2884 (2009). doi:10.1172/JCI40839.
Primary trauma to the CNS initiates a series of interrelated responses, including edema, excitotoxicity, and inflammation, that lead to secondary injury, resulting in further expansion of the initial lesion and additional loss of neurological function. The treatment of neuroinflammation in the context of both traumatic brain injury and spinal cord injury (SCI) still lacks a standard, universally accepted therapy that leads to improved neurological outcomes. There exists a clear, unmet medical need for an effective antiinflammatory treatment for the acute and chronic stages of traumatic brain injury and SCI arising in general civilian as well in injured military personnel populations. Currently, an important area of SCI research focuses on 


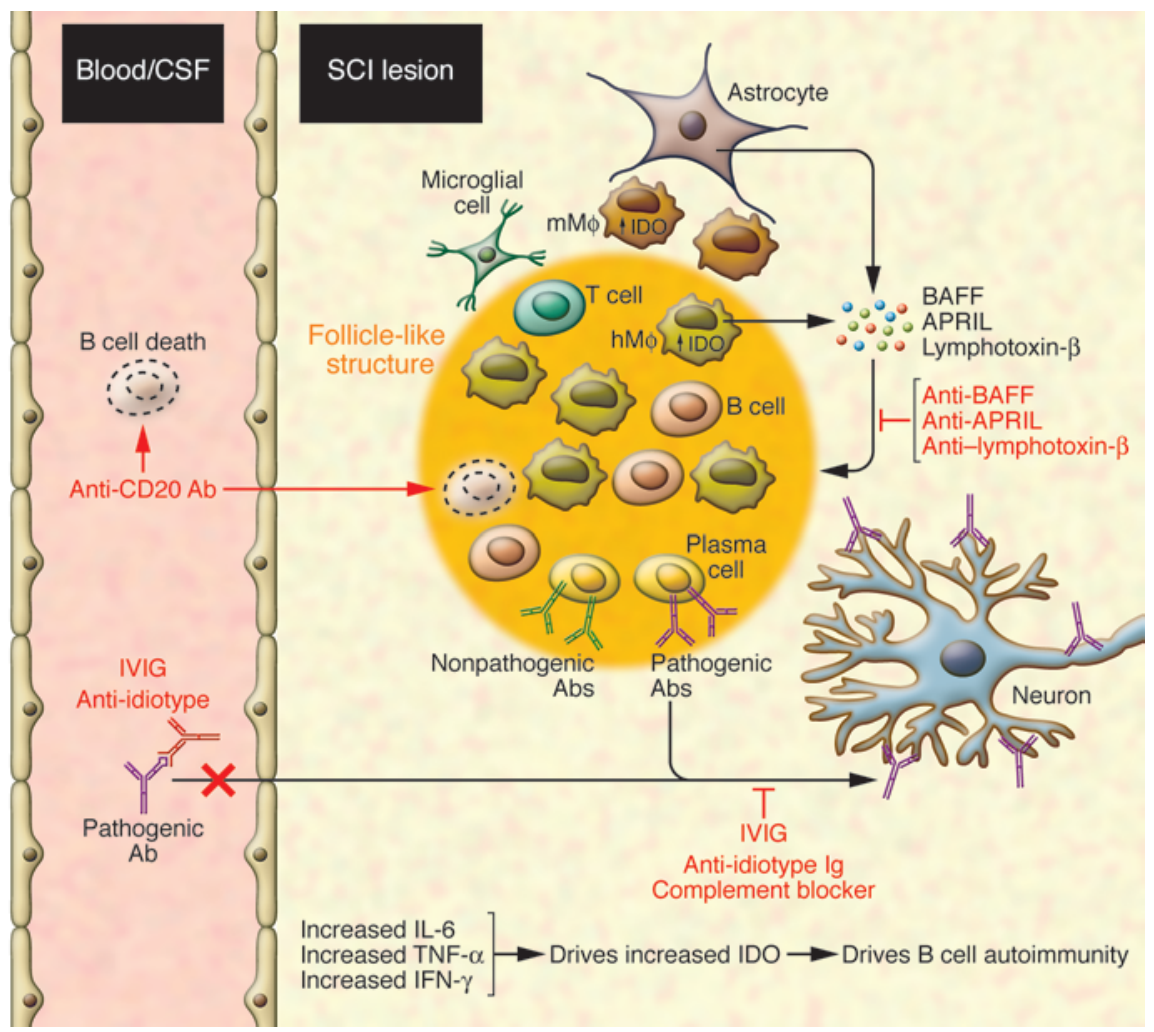

Figure 1

$\mathrm{B}$ cells and an autoimmune response contribute to poor neurological outcome following SCI. After SCl, B cells, together with other leukocytes such as monocyte/macrophages (hM $\phi)$ and $T$ cells, infiltrate the lesion. Resident activated microglia, microglia-derived macrophages $(\mathrm{mM} \phi)$, and astrocytes, also surround and/or infiltrate the lesion. Infiltrating macrophages as well as proliferating astrocytes respond to the $\mathrm{SCI}$ by producing BAFF, Iymphotoxin- $\beta$, and APRIL, which support B cell survival, proliferation, and differentiation as well as B cell follicle development. Follicle-like structures appear at the lesion area and house activated B cells and plasma cells. Additionally, the high levels of IFN- $\gamma$ and TNF- $\alpha$ present at the SCI lesion will induce IDO expression, a key regulator of tolerance and a preventer of autoimmunity. IDO is expressed by activated microglia and infiltrating leukocytes. The toxic tryptophan metabolites generated by IDO render T cells but not B cells highly susceptible to apoptosis. This creates a cytokine environment that favors B cell autoimmunity and antibody production. Thus, within the $\mathrm{SCl}$ lesion, the local environment is conducive to the support of $\mathrm{B}$ cells and antibody production. Therapeutics such as blockers of BAFF, APRIL, or lymphotoxin- $\beta$; anti-CD20 monoclonal antibodies; and IVIG could prevent follicle-germinal center development, deplete B cells (but not plasma cells), and block the pathogenic activity of autoantibodies to neural antigens at the site of the SCl. These therapeutic agents could be used singly or in combination in order to obtain maximal benefit.

understanding the dual nature of postSCI neuroinflammation. It can lead to increased damage to the neural tissue, yet it is an essential part of the wound healing process $(1,2)$. It is becoming increasingly clear that injury to the CNS results in alterations to the systemic immune system that in turn affect the competing pathogenic and wound healing processes evolving at the site of injury (3-5). The study reported by Ankeny et al. in this issue of the JCI (6) offers a new perspective on a poorly understood aspect of CNS inflammation in response to traumatic injury in mice, namely the impact of B cells and autoimmunity on neurological outcomes. This new understanding of SCI disease pathogenesis, if confirmed in humans, reveals avenues for the development of novel neuroprotective immunotherapies.

\section{$B$ cells in the normal and diseased CNS}

B lymphocytes are a key component of the adaptive immune system. They arise from bone marrow hematopoietic stem cells that differentiate in an antigen-independent manner to the immature B cell stage. In the presence of antigen, further differentiation leads to mature and activated B cells. Activated B cells convert into short-lived, antibody-secreting plasma cells or into memory $B$ cells and then into long-lived, antibodysecreting plasma cells $(7,8)$. Activated $B$ cells and long-lived plasma cells migrate not only to the bone marrow and secondary lymphoid organs but to the CNS via normal homeostatic processes (7). This $\mathrm{B}$ cell recruitment mechanism is upregulated during CNS autoimmune diseases, such as MS $(7,9)$. There are several B cellspecific factors and receptor interactions that are critical to $\mathrm{B}$ cell function and are potential therapeutic targets. B cell-activating factor (BAFF), lymphotoxin- $\beta$, and a proliferation-inducing ligand (APRIL) have roles important to B cell survival, differentiation, germinal center formation, and antibody synthesis $(7,8)$. These factors are secreted by macrophages and, within the CNS, by astrocytes $(7,8)$. Thus, B cells have an established mechanism that allows them to traffic to and be supported in the CNS. The normally assumed role of B cells is to produce antibodies, but it is now clear that B cells can serve as potent regulatory and antigen-presenting cells $(8,10)$. It is well known that under normal circumstances human cerebrospinal fluid (CSF) harbors low levels of antibody, produced by longlived plasma cells, some of which are autoreactive (11). The role of $\mathrm{B}$ cells in various CNS autoimmune conditions is also well known. However, until recently the role of B cells was deemed secondary to that of $\mathrm{T}$ cells in disease pathogenesis. There is now clear evidence that B cells and associated autoantibodies can play an important primary role in CNS autoimmune disease $(8,12)$.

\section{SCI leads to pathogenic autoantibody production}

The results presented in this issue by Ankeny et al. (6) clearly demonstrate that, in a mouse model of SCI, trauma of moderate severity at thoracic level 9 (T9) leads to a surprisingly robust $B$ cell response that produces pathogenic antibodies. This important conclusion is supported by experiments demonstrating that spontaneous neurological recovery after injury was greatly improved in B cell-knockout mice compared with WT mice. Following SCI, coordinated stepping involving all four limbs was achieved in $88 \%$ of B cell-knockout mice but in only $35 \%$ of WT mice at the 
end of the nine-week observation period. Consistent with this improved functional recovery, the neuropathology observed in the B cell-knockout mice was also markedly less pronounced compared with WT animals. This suggests that, in WT mice that received an SCI, B cells play a role in the evolving inflammatory response that impedes neurological recovery. Importantly, passive transfer (injection) of purified pathogenic antibody into the spinal cord of WT mice under sterile conditions induced a similar type of neurotoxicity to that observed in mice with SCI. This confirmed that neurotoxic product of the SCIinduced B cell activation was likely pathogenic antibodies.

This article (6) raises the question as to why B cells produce pathogenic antibodies when the SCI is in the lower half of the spinal cord (T9-T10). Yet, these same authors previously reported that when the SCI occurs at a higher level (T4-T5) profound immune suppression occurs, including that of B cell function (13). A likely explanation may lie in the fact that a high SCI disrupts the cholinergic antiinflammatory pathway by removing the sympathetic contribution (via the splenic nerves) due to injury to the intermediolateral column sympathetic fibers. This pathway plays a key role in regulating systemic inflammation (4). Further investigation is required to understand the underlying pathological mechanisms created by the loss of sympathetic control that results in immune suppression and whether this is a permanent feature of SCI located above T4/T5. Alternatively, the appearance of autoimmune neuropathogenic antibodies may only be delayed.

The presence of pathogenic antibodies in the spinal lesion is in part derived from systemic sources via a compromised bloodspinal cord barrier after injury. However, evidence was presented by Ankeny et al. (6) that B cell "follicle-like" structures were present in the lesion area, suggesting that local antibody production may be critical to the pathogenic outcome. Both activated $B$ cells and plasma cells are present in these structures. The presence of B cell follicle-like structures in the diseased CNS is not unique to SCI but has been described in other CNS autoimmune diseases, including MS (9). The local SCI inflammatory environment likely would support the development of B cell follicle-like structures in two ways. Infiltrating monocyte/macrophages as well as the large number of proliferating astrocytes responding to the SCI likely could sup- ply sufficient BAFF and APRIL to support B cell survival, proliferation, differentiation as well as follicle development (Figure 1). Secondly, SCI-induced neuroinflammation dysregulates an important immune regulatory mechanism known as the tryptophan-kynurenine pathway (14-17). The critical component of this tryptophan catabolic pathway is the rate-limiting enzyme, indoleamine 2,3-dioxygenase (IDO), which is primarily induced by IFN- $\gamma$ but can be superinduced in the presence of TNF- $\alpha$ $(14,15)$. Both cytokines are present following SCI. IDO is a key regulator of tolerance and a preventer of autoimmunity $(18,19)$. IDO is expressed by activated microglia and infiltrating leukocytes (14). T cells, but not $B$ cells, are particularly sensitive to the effects of IDO. The tryptophan metabolites generated by IDO, such as quinolinic acid, are well known neurotoxins and also render $\mathrm{T}$ cells, but not B cells, highly susceptible to apoptosis and other aspects of IDO-mediated regulation $(4,14,17,19)$. This alters the local cytokine environment to one that favors B cell autoimmunity and antibody production $(17,19)$ (Figure 1). Thus, within the SCI lesion, the local environment is conducive to the support of B cells and antibody production.

\section{Potential new immunotherapies for $\mathrm{SCl}$}

The results reported by Ankeny et al. (6) suggest opportunities for the development of new immunotherapies for SCI. Therapies that target the B cell or block the affects of pathogenic antibodies have demonstrated considerable promise in early phase I/II clinical trials $(7,10,20)$. However, there are no FDA-approved, licensed anti-B cell products yet available for CNS autoimmune indications. Plasmapheresis is one approach that might improve neurological outcomes in SCI patients, by removing pathogenic autoantibodies at crucial times in the early stages of recovery, leading to a potential neuroprotective benefit. Whether plasmapheresis would lead to sustained improved neurological outcomes for SCI patients needs to be determined. Intravenous immunoglobulin (IVIG) administration has the potential for significant neuroprotection. IVIG is known to block, via an anti-idiotype mechanism, the binding of pathogenic antibodies to their targets, and IVIG also blocks the complement system (20) (Figure 1). The results of Ankeny et al. (6) support the testing of IVIG for SCI treatment, as both autoantibody binding to spinal tissue and complement were demonstrated to be involved in SCI-associated autoantibody-induced pathology. IVIG therapy is generally well tolerated with few adverse side effects (20).

$B$ cell depletion as a neuroprotective therapy is another viable option for SCI (Figure 1). Clinical trial results testing the beneficial affects of B cell depletion in various CNS autoimmune conditions support the approach, but with caution $(7,10,12)$. Anti-CD20 antibody-mediated depletion of B cells is fast, effective, long-lasting, and largely free of adverse events in shortterm applications $(7,10)$. Anti-CD20 does not block anamnestic antibody-mediated immune responses and is not immunosuppressive in that context (8). Long-term application of anti-CD20 therapy is still a concern (8) especially since SCI individuals are already immunosuppressed (3). For SCI, the real issue is whether anti-CD20 antibody-mediated B cell depletion will lead to improved outcomes. CD20 is expressed on early, mature, and activated B cells but not on antibody-secreting plasma cells (8). Consequently, long-term B cell depletion only has small to moderate affects on reducing plasma antibody levels and is not effective in eliminating B cells in follicles $(8,12)$. Pre-existing plasma cells that secret potentially pathogenic antibodies will not be eliminated by anti-CD20 therapy. It may be affective in preventing the appearance of de novo-created short-lived plasma cells by elimination of their precursors $(7,8)$. If antigen presentation or other B cell functions are critical to the induction of the SCIinduced pathogenic autoantibodies, then anti-CD20 therapy will likely be effective. These are important issues that need to be resolved. Alternatively, there are monoclonal antibody therapies being developed to block the actions of BAFF, lymphotoxin- $\beta$, and APRIL (8). Providing sufficient levels of neutralizing antibody could be delivered to target areas, preventing the development of B cell follicles in the SCI lesion area and in secondary lymphoid tissues may reduce the levels of autoantibody, such that the pathogenic consequences are minimal.

It remains to be determined whether pathogenic autoantibodies are a primary cause of human secondary SCI. However, there is now sufficient evidence to support the development of strategies, in appropriate animal models, to test whether potential immunotherapies restricting B cell responses following SCI warrant translation to the treatment of human SCI. 


\section{Acknowledgments}

We thank Lynne C. Weaver for her critical review of the literature and Ilda Moniz for her assistance.

Address correspondence to: Gregory A. Dekaban, BioTherapeutics Research Laboratory, Molecular Brain Research Group, Robarts Research Institute, Rm 2-12, 100 Perth Drive, London, Ontario, N6A 5K8 Canada. Phone: (519) 663-5777 ext. 24241; Fax: (519) 391-5789; E-mail: dekaban@robarts.ca.

1. Rossignol, S., Schwab, M., Schwartz, M., and Fehlings, M.G. 2007. Spinal cord injury: time to move? J. Neurosci. 27:11782-11792

2. Popovich, P.G., and Longbrake, E.E. 2008. Can the immune system be harnessed to repair the CNS? Nat. Rev. Neurosci. 9:481-493.

3. Riegger, T., et al. 2009. Immune depression syndrome following human spinal cord injury (SCI): a pilot study. Neuroscience. 158:1194-1199.

4. Rosas-Ballina, M., and Tracey, K.J. 2009. Cholinergic control of inflammation. J. Intern. Med. 265:663-679.
5. Lu, J., et al. 2009. Systemic inflammatory response following acute traumatic brain injury. Front. Biosci. 14:3795-3813

6. Ankeny, D.P., Guan, Z., and Popovich, P.G. 2009 $B$ cells produce pathogenic antibodies and impair recovery after spinal cord injury in mice. J. Clin. Invest. 119:2990-2999.

7. Dalakas, M.C. 2008. B cells as therapeutic targets in autoimmune neurological disorders. Nat. Clin. Pract. Neurol. 4:557-567.

8. Dalakas, M.C. 2008. Invited article: inhibition of B cell functions: implications for neurology. Neurology. 70:2252-2260

9. Corcione, A., et al. 2004. Recapitulation of B cell differentiation in the central nervous system of patients with multiple sclerosis. Proc. Natl. Acad. Sci.U. S. A. 101:11064-11069.

10. Waubant, E. 2008. Spotlight on anti-CD20. Int. MS J. 15:19-25.

11. Elkon, K., and Casali, P. 2008. Nature and functions of autoantibodies. Nat. Clin. Pract. Rheumatol. 4:491-498.

12. Matsushita, T, and Tedder, T.F. 2009. B-lymphocyte depletion for the treatment of multiple sclerosis: now things really get interesting. Expert Rev. Neurother. 9:309-312.

13. Lucin, K.M., Sanders, V.M., Jones, T.B., Malarkey, W.B., and Popovich, P.G. 2007. Impaired antibody synthesis after spinal cord injury is level dependent and is due to sympathetic nervous system dysregulation. Exp. Neurol. 207:75-84.

14. Kwidzinski, E., and Bechmann, I. 2007. IDO expression in the brain: a double-edged sword. J. Mol. Med. 85:1351-1359.

15. Oxenkrug, G.F. 2007. Genetic and hormonal regulation of tryptophan kynurenine metabolism: implications for vascular cognitive impairment, major depressive disorder, and aging. Ann. N. Y. Acad. Sci. 1122:35-49.

16. Kwidzinski, E., et al. 2005. Indolamine 2,3-dioxygenase is expressed in the CNS and down-regulates autoimmune inflammation. FASEB J. 19:1347-1349.

17. Xu, H., Zhang, G.X., Ciric, B., and Rostami, A. 2008. IDO: a double-edged sword for $\mathrm{T}(\mathrm{H}) 1 / \mathrm{T}(\mathrm{H}) 2$ regulation. Immunol. Lett. 121:1-6.

18. Mellor, A.L., and Munn, D.H. 2008. Creating immune privilege: active local suppression that benefits friends, but protects foes. Nat. Rev. Immunol. 8:74-80.

19. Scott, G.N., et al. 2009. The immunoregulatory enzyme IDO paradoxically drives B cell-mediated autoimmunity. J. Immunol. 182:7509-7517.

20. Gold, R., Stangel, M., and Dalakas, M.C. 2007. Drug insight: the use of intravenous immunoglobulin in neurology--therapeutic considerations and practical issues. Nat. Clin. Pract. Neurol. 3:36-44.

\title{
Reduced thymus activity and infection prematurely age the immune system
}

\author{
Ronald E. Gress ${ }^{1}$ and Steven G. Deeks ${ }^{2}$
}

1Experimental Transplantation and Immunology Branch, Center for Cancer Research, National Cancer Institute Bethesda, Maryland, USA. ${ }^{2}$ UCSF, San Francisco, California, USA.

The aging process affects all aspects of the immune system, particularly the $T$ cells. The immune system in older individuals is often characterized by lower $T$ cell numbers, lower naive/memory $T$ cell ratios, and lower $T$ cell diversity. Most measures of inflammation increase with age. Why this happens, and why there is so much person-to-person variability in these changes, is not known. In this issue of the JCI, Sauce and colleagues show that removal of the thymus during infancy results in premature onset of many of these age-associated changes to the immune system (see the related article beginning on page 3070 ). The effect of thymectomy was particularly notable in those individuals who acquired CMV infection. Data from this study, as well as data from other observational settings, suggest that reduced thymic function and persistent viral infections combine to accelerate a decline in immunologic function.

The aging process affects all organs, including the immune system. Immunologic aging, generally referred to as "immunosenescence," clearly affects $\mathrm{T}$ cell function, but changes also occur in B cells, antigenpresenting cells, NK cells, and perhaps even

Conflict of interest: The authors have declared that no conflict of interest exists.

Citation for this article: J. Clin. Invest. 119:2884-2887 (2009). doi:10.1172/JCI40855. stem cells. With respect to T cells, the major age-associated changes include a decline in the total number of cells, a shift from a naive to a memory/effector $\mathrm{T}$ cell population, and a decline in $\mathrm{CD}^{+} \mathrm{T}$ cell receptor repertoire diversity. Generalized inflammation also increases with age, perhaps due to a loss of immunoregulatory function. These age-associated alterations result in immune compromise with potential susceptibilities to infection, autoimmunity, and neoplasia. Nearly all of the typical ageassociated complications, including heart disease, cancer, infection, dementia, and frailty, have been epidemiologically linked (but not necessarily causally linked) to the process of immunosenescence.

\section{Effect of thymectomy and CMV on immunosenescence}

Although the pathogenesis of immunosenescence has been well studied in experimental models, very little mechanistic work has been performed in humans. In this issue of the JCI, Sauce and colleagues studied a group of young adults who as infants underwent complete removal of their thymus during the surgical correction of a congenital heart defect (1). Compared with age-matched controls, the thymectomized adults had a number of classic age-associated immunologic abnormalities, including lower total $\mathrm{T}$ cell counts and a preferential loss of naive $\mathrm{T}$ cells. These observations were not surprising, because the thymus is an estab- 